\title{
Banding Pattern Indicative of Echinococcosis in a Commercial Cysticercosis Western Blot
}

\author{
D. Tappe ${ }^{1}$, B. Grüner ${ }^{2}$, P. Kern², M. Frosch ${ }^{1}$ \\ ${ }^{1}$ Institute of Hygiene and Microbiology, University of Würzburg, Germany \\ ${ }^{2}$ Comprehensive Infectious Diseases Center, Division of Infectious Diseases and Clinical Immunology, University Hospital and \\ Medical Center Ulm, Germany
}

\begin{abstract}
Objective: A commercial cysticercosis Western blot was evaluated for serological cross-reactivity of sera from patients with alveolar (AE) and cystic echinococcosis (CE).

Methods: A total of 161 sera were examined, including 31 sera from AE-patients, 11 sera from CE-patients, 9 sera from patients with other parasitic diseases and 109 sera from patients with unrelated medical conditions. All AE- and CE-sera were also examined by the echinococcosis Western blot.

Results: More sera from patients with AE than with CE showed cross-reactivity in the form of ladder-like patterns ("Mikado aspect") and untypical bands at 6-8 $\mathrm{kDa}(71 \%$ and $77.4 \%$ versus $27.3 \%$ and $45.5 \%$, respectively). In contrast, triplets of bands in the area above $50 \mathrm{kDa}$ and between 24 and $39-42 \mathrm{kDa}$ were more frequent in $\mathrm{CE}$ than in $\mathrm{AE}$ sera. The fuzzy band at 50-55 kDa typical for cysticercosis was absent in all $\mathrm{AE}$ and CE sera.

Conclusions: Atypical banding patterns in the cysticercosis Western blot should raise the suspicion of a metacestode infection different from Taenia solium, i.e. Echinococcus multilocularis or E. granulosus, especially when the Mikado aspect and an altered 6-8 $\mathrm{kDa}$ band is visible in the absence of a fuzzy 50-55 $\mathrm{kDa}$ band.
\end{abstract}

Key words: Alveolar echinococcosis, Cystic echinococcosis, Cysticercosis, Western blot, Banding pattern, Serology, Cross-reactivity

\section{INTRODUCTION}

Like cysticercosis, alveolar echinococcosis (AE) and cystic echinococcosis (CE) may affect the central nervous system (CNS) and subcutaneous tissue. In contrast to cysticercosis however, CNS and skin involvement in echinococcosis, especially AE [1] is rare. Still, the multivesicular appearance of the racemose cysticercus in the CNS may be confused with either form of echinococcosis. A peculiar banding pattern seen on a cysticercosis Western blot in a patient with cerebral AE [2] prompted us to further investigate cross-reactivity of $\mathrm{AE}$ and $\mathrm{CE}$ sera on a commercially available cysticercosis Western blot.

\section{Materials AND Methods}

Sera: A total of 161 sera from 161 patients were examined in the cysticercosis Western blot. Among these, 31 sera were from patients with parasitologically proven AE, 11 sera were from patients with $\mathrm{CE}$ and 1 serum was obtained from a patient with cysticercosis. 3 sera from patients with each schistosomiasis, toxocariasis and trichinellosis, respectively, were also tested. 109 serum samples were derived from patients with unrelated medical conditions. All sera from patients with $\mathrm{AE}$ and $\mathrm{CE}$ were also examined with the echinococcosis Western blot.

Cysticercosis and Echinococcosis Western blots: The Cysticercosis Western Blot IgG and the Echinococcus Western Blot IgG (both LDBIO Diagnostics, Lyon, France) were used according to the manufacturer's instructions.

\section{RESUlts AND Discussion}

To our knowledge, no systematic investigation on cross-reactivity of AE sera on cysticercosis blots has been reported. Recently, cross-reactivity of CE sera on an enzyme-linked immunoelectrotransfer blot (EITB) for cysticercosis has been published [3]. In our study, none of the AE and CE sera exhibited a pattern typical for cysticercosis. Instead, cross-reactivity in the form of ladder-like patterns ("Mikado aspect" [4]), untypical bands at $6-8 \mathrm{kDa}$ and the absence of a fuzzy band at $50-55 \mathrm{kDa}$ were observed.

The Mikado aspect was shown in 22 out of $31 \mathrm{AE}$ sera $(71 \%)$ and in only 3 out of $11 \mathrm{CE}$ sera $(27.3 \%)$ (Fig. 1). This pattern was also seen in 2 out of 3 sera from patients with schistosomiasis, in 1 out of $3 \mathrm{pa}-$ tients with toxocariasis and in 4 out of 109 patients with unrelated diseases (1 patient with a brain abscess, borreliosis, idiopathic eosinophilia and psychosis each, respectively). According to the manufacturer [4], this non-specific binding pattern may concern only one part of the blot strip and was shown as an example in sera of 1 patient with $\mathrm{CE}$ and 2 patients with $\mathrm{AE}$. No frequencies in echinococcosis patients are reported, however.

In this study, 24 out of $31 \mathrm{AE}$ sera showed a narrow single or double band at $6-8 \mathrm{kDa}$ in the cysticercosis 


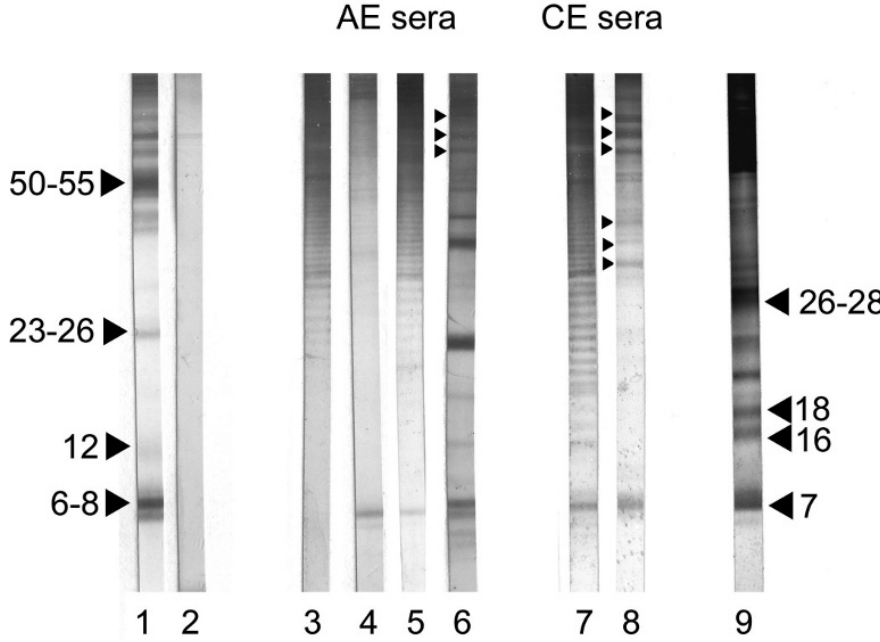

Fig. 1. Examples of cross-reactivity profiles in sera of patients with alveolar and cystic echinococcosis tested in the cysticercosis Western blot.

Strip1 1, cysticercosis positive control. Molecular size markers in $\mathrm{kDa}$ are shown. The band at $12 \mathrm{kDa}$ is absent in this serum. Strip 2, cysticercosis negative control. Strip 3, AE serum with Mikado-aspect only. Strip 4, AE serum with band at approx. $7 \mathrm{kDa}$. Strip 5, AE serum with both Mikado-aspect and $7 \mathrm{kDa}$-band. Strip 6, AE serum which demonstrated the T1 triplet (small triangles) and various other bands. Strip 7, CE serum with both Mikado-aspect and $7 \mathrm{kDa}$-band. Strip 8, CE serum with $7 \mathrm{kDa}$-band and triplets T1 (small triangles above $50-55 \mathrm{kDa}$ ) and T2 (small triangles below $40 \mathrm{kDa}$ ). Strip 9, Echinococcus Western blot positive control with AE serum. Molecular size markers in $\mathrm{kDa}$ are shown. Abbreviations: AE, alveolar echinococcosis; CE, cystic echinococcosis
Western blot (77.4\%). A corresponding large single band at $7 \mathrm{kDa}$ was present in all these sera when tested by the echinococcosis Western blot (Fig. 1). In the remaining 7 AE sera, both the $6-8 \mathrm{kDa}$ and the $7 \mathrm{kDa}$ band in the cysticercosis and echinococcosis Western blot were absent, respectively. In patients with $\mathrm{CE}$, a single or double $6-8 \mathrm{kDa}$ band was present in only 5 out of 11 sera when tested in the cysticercosis blot $(45.5 \%)$, but all 11 sera showed the $7 \mathrm{kDa}$ band in the echinococcosis blot. Bands at $6-8 \mathrm{kDa}$ were absent in all sera from patients with schistosomiasis, toxocariasis, trichinellosis and unrelated medical conditions in the cysticercosis blot. Much lower frequencies of $50 \%$ and $10 \%$ for the presence of the $6-8 \mathrm{kDa}$ band in $\mathrm{AE}$ and $\mathrm{CE}$ sera, respectively, were shown by the manufacturer [4]. In all AE and $\mathrm{CE}$ sera that cross-reacted with the $6-8 \mathrm{kDa}$ band in the cysticercosis blot, the band corresponded to the $7 \mathrm{kDa}$ band in the echinococcosis blot. This low molecular-weight band could thus be tapeworm-specific. A combination of both the Mikado aspect and the presence of an atypical band at $6-8 \mathrm{kDa}$ was detected in $58.1 \%$ of AE sera and in $27.3 \%$ of CE sera.

The so-called T1-triplet of bands above a $50 \mathrm{kDa}$ band seen in $78 \%$ of CE sera tested on an EITB for cysticercosis as decribed by van Doorn et al. [3] were not readily identified in our study. However, 2-3 similar bands directly above the $50-55 \mathrm{kDa}$ area were seen in 17 out of $31 \mathrm{AE}$ sera $(54.8 \%)$ and in 10 out of 11 CE sera $(90.9 \%)$ in our survey. These 3 bands were also visible in the presence of the fuzzy $50-55 \mathrm{kDa}$ band on the control strip incubated with a cysticercosis positive serum. In all $\mathrm{AE}$ and $\mathrm{CE}$ sera, however, the $50-55 \mathrm{kDa}$ band itself was absent (Fig. 1). In van Doorn's study [3], a $50 \mathrm{kDa}$ band was present in cysticercosis sera, but also absent in many, if not all CE sera tested (no concise data published). The so-called T2-triplet of bands between 24 and 39-42 kDa, which was infrequently detected in CE sera by EITB [3] was not found in any of our AE sera, but in $7 \mathrm{CE}$ sera (Fig.1). Although the EITB and the Western blot use different methodology, a concordance of $98 \%$ was shown for these techniques [5].

In conclusion, atypical banding patterns in the cysticercosis Western blot should be interpreted with caution and should raise the suspicion of a metacestode infection different from Taenia solium in conjunction with the clinical status of the patient. The Mikado aspect may possibly indicate an infection with a metazoan parasite other than $T$. solium. The presence of an altered $6-8 \mathrm{kDa}$ band in the absence of a fuzzy 50-55 $\mathrm{kDa}$ band could signal a larval infection with a different cestode than T. solium, i.e. E. multilocularis or E. granulosus. A combination of these blot patterns further favours the diagnosis of a possible echinococcal infection. Different serological assays with a higher specificity should then be performed.

Acknowledgments: The authors would like to thank Mechthild Schulze, Würzburg, for excellent technical assistance.

\section{REFERENCES}

1. Kern P, Bardonnet K, Renner E, Auer H, Pawlowski Z, Ammann RW, Vuitton DA, Kern P. European echinococcosis registry: human alveolar echinococcosis, Europe, 1982-2000. Emerg Infect Dis. 2003; 9: 343-349.

2. Tappe D, Weise D, Ziegler U, Müller A, Müllges W, Stich A. Brain and lung metastasis of alveolar echinococcosis in a refugee from a hyperendemic area. J Med Microbiol. 2008; 57: 1420-1423.

3. van Doorn HR, Wentink-Bonnema E, Rentenaar RJ, van Gool T. Specific cross-reactivity in sera from cystic echinococcosis patients in an enzyme-linked immunoelectrotransfer blot for cysticercosis diagnostics. Trans R Soc Trop Med Hyg. 2007; 101: 948-950.

4. LDBIO Diagnostics: Cysticercosis Western Blot IgG. Instructions for use. F163-CYSWBG-a-25/08/2005 v6. 2005.

5. Villota GE, Gomez DI, Volcy M, Franco AF, Cardona EA, Isaza R, Sanzón F, Teale JM, Restrepo BI. Similar diagnostic performance for neurocysticercosis of three glycoprotein preparations from Taenia solium metacestodes. Am J Trop Med Hyg. 2003; 68: 276-280.

Received: June 17, 2009 / Accepted: July 27, 2009

Address for correspondence:

Dennis Tappe

Institute of Hygiene and Microbiology

University of Würzburg,

Josef-Schneider-Str. 2

97080 Würzburg

Germany

Tel.: $\quad+49-931-201-46036$

Fax: +49-931-201-46445

E-mail: dtappe@hygiene.uni-wuerzburg.de 